\title{
AUTOMATED MULTI-LEVEL SUBSTRUCTURING FOR NONLINEAR EIGENPROBLEMS
}

\author{
K. Elssel and H. Voss \\ Section of Mathematics, \\ Hamburg University of Technology, Hamburg, Germany
}

\begin{abstract}
In this paper we generalize the automated multi-level substructuring method to certain classes of nonlinear eigenvalue problems which can be partitioned into an essential linear and positive definite pencil and a small residual. The efficiency of the method is demonstrated by numerical examples modeling damped vibrations of a structure with nonproportional damping, a gyroscopic eigenproblem, and a rational eigenproblem governing free vibrations of a fluid-solid structure.
\end{abstract}

Keywords: automated multi-level substructuring, AMLS, nonlinear eigenproblem, eigenvalue, eigenvector, sparse matrix, iterative projection method, Arnoldi method

\section{Introduction}

In this paper we consider the nonlinear eigenvalue problem

$$
T(\lambda) x=0
$$

where $T(\lambda) \in \mathbb{C}^{n \times n}$ is a family of large and sparse matrices depending on a parameter $\lambda \in D \subset \mathbb{C}$. As in the linear case $T(\lambda)=\lambda B-A$ a parameter $\lambda$ is called an eigenvalue of $T(\cdot)$ if problem (1) has a nontrivial solution $x \neq 0$ which is called a corresponding eigenvector.

Problems of this type arise in damped vibrations of structures, vibrations of rotating structures, stability of linear systems with retarded argument, lateral buckling problems, vibrations of fluid-solid structures, or numerical simulation of quantum dot heterostructures, to name just a few.

Over the last few years, a new method for huge linear eigenvalue problems

$$
K x=\lambda M x
$$


where $K \in \mathbb{C}^{n \times n}$ and $M \in \mathbb{C}^{n \times}$ are Hermitian and positive definite, known as $A u$ tomated Multi-Level Substructuring (AMLS), has been developed by Bennighof and co-authors, and has been applied to frequency response analysis of complex structures $[3,4,5,6]$. Here the large finite element model is recursively divided into very many substructures on several levels based on the sparsity structure of the system matrices. Assuming that the interior degrees of freedom of substructures depend quasistatically on the interface degrees of freedom, and modeling the deviation from quasistatic dependence in terms of a small number of selected substructure eigenmodes the size of the finite element model is reduced substantially yet yielding satisfactory accuracy over a wide frequency range of interest. Recent studies ([15, 17, 22], e.g.) in vibroacoustic analysis of passenger car bodies where very large FE models with more than six million degrees of freedom appear and several hundreds of eigenfrequencies and eigenmodes are needed have shown that AMLS is considerably faster than Lanczos type approaches.

From a mathematical point of view, AMLS is nothing else but a projection method where the large problem under consideration is projected to a subspace spanned by a small number of eigenmodes of undamped clamped substructures on several levels. With respect to this basis the projection of the stiffness matrix $K$ becomes diagonal, and the mass matrix $M$ is projected to a generalized arrowhead form.

In this presentation we discuss the generalization of AMLS to the nonlinear eigenvalue problem (1). To this end we identify an essential linear part of the nonlinear eigenproblem, i.e. we rewrite problem (1) as

$$
T(\lambda)=K x-\lambda M x+R(\lambda) x=0
$$

where $K \in \mathbb{C}^{n \times n}$ and $M \in \mathbb{C}^{n \times n}$ are Hermitian and positive definite matrices, and $R(\lambda) \in \mathbb{R}^{n \times n}$ is small. We construct the ansatz space for the projection by the AMLS approach using the matrices $K$ and $M$ only, and apply all transformations and projections to the nonlinear part $R(\cdot)$ simultaneously. Thus, we obtain a nonlinear eigenproblem of the same type as (1) but of much smaller dimension which can be solved by any appropriate method, i.e. by a dense solver if the projected problem is small, by linearization if the underlying problem is a polynomial eigenproblem, or by an iterative projection method of Arnoldi [26] or Jacobi-Davidson type [27].

The efficiency of the method is demonstrated by three classes of problems, a gyroscopic eigenproblem modeling a rotating tire, a rational eigenvalue problem

$$
K x+\omega^{2} M x-\sum_{j=1}^{p} \frac{1}{1+b_{j} \omega} \Delta K_{j} x=0
$$

modeling the damped vibrations of a structure with nonproportional damping where a viscoelastic constitutive relation is assumed to describe the behavior of the material, and a rational eigenvalue problem governing free vibrations of fluid-solid structure. 


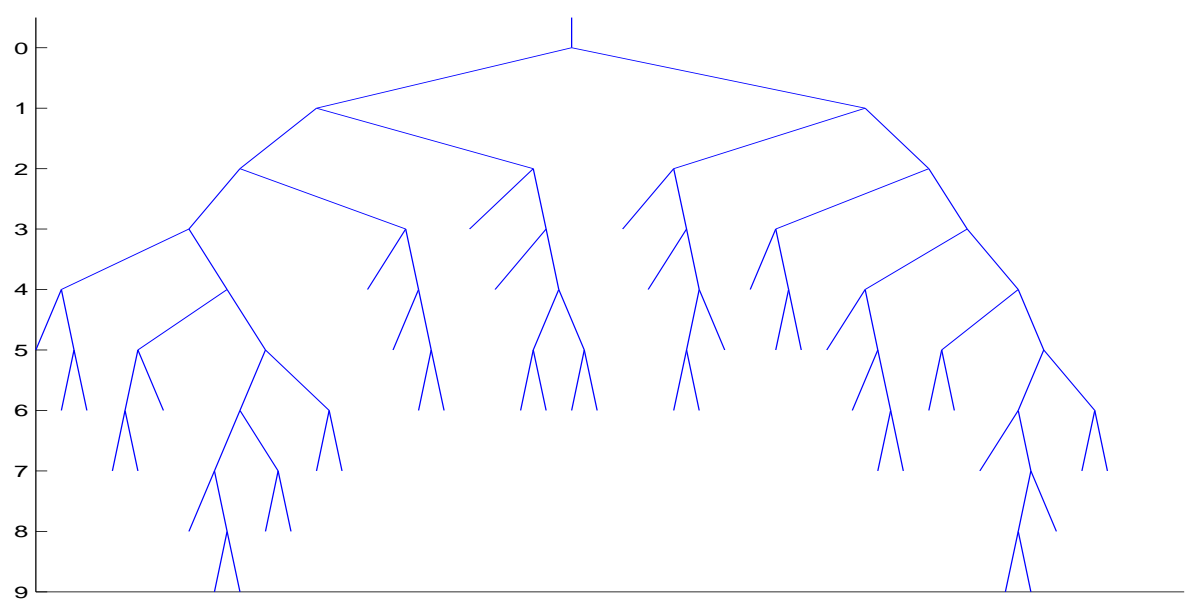

Fig. 1: Substructure tree

\section{AMLS for linear eigenproblems}

In this section we summarize the Automated Multi-Level Substructuring (AMLS) method for the linear eigenvalue problem

$$
K x=\lambda M x
$$

which was developed by Bennighof and co-workers over the last few years $[4,6,15]$, who applied it to solve frequency response problems involving large and complex models. Here, $K$ is the stiffness matrix and $M$ the mass matrix of a finite element model of a structure.

Similarly as in the component mode synthesis (CMS) the structure is partitioned into a small number of substructures based on the sparsity pattern of the system matrices, but more generally than in CMS these substructures in turn are substructured on a number of levels yielding a tree topology for the substructures. Fig. 1 shows an example were each substructure has at most two children.

We stress the fact that substructuring does not mean that it is obtained by a domain decomposition of a real structure, but it is understood in a purely algebraic sense, i.e. the dissection of the matrices can be derived by applying a graph partitioner like CHACO [13] or METIS [16] to the matrix under consideration. However, because of its pictographic nomenclature we will use terms like substructure or eigenmode from frequency response problems when describing the AMLS method.

Substructures on the lowest level consist of a small number of degrees of freedom, which are partitioned into two sets: interface degrees of freedom which are shared with an adjacent substructure, and interior or local degrees of freedom which are only connected to degrees of freedom in their own substructure. To demonstrate the AMLS method we distinguish only between two substructures on the lowest level (corresponding to index sets $I_{1}$ and $I_{2}$ ), the interface between these substructures (with index set $I_{3}$ ) and the remaining degrees of freedom (index set $I_{4}$ ). Partitioning the displacement vector $x$ correspondingly and reordering the matrices $K$ and $M$ the eigenvalue 
problem (5) obtains the following form

$$
\left(\begin{array}{cccc}
K_{11} & O & K_{13} & K_{14} \\
O & K_{22} & K_{23} & K_{24} \\
K_{13}^{T} & K_{23}^{T} & K_{33} & K_{34} \\
K_{14}^{T} & K_{24}^{T} & K_{34}^{T} & K_{44}
\end{array}\right)\left(\begin{array}{l}
x_{1} \\
x_{2} \\
x_{3} \\
x_{4}
\end{array}\right)=\lambda\left(\begin{array}{cccc}
M_{11} & O & M_{13} & M_{14} \\
O & M_{22} & M_{23} & M_{24} \\
M_{13}^{T} & M_{23}^{T} & M_{33} & M_{34} \\
M_{14}^{T} & M_{24}^{T} & M_{34}^{T} & M_{44}
\end{array}\right)\left(\begin{array}{l}
x_{1} \\
x_{2} \\
x_{3} \\
x_{4}
\end{array}\right) .
$$

To remove the off-diagonal blocks $K_{i j}, i=1,2, j=3,4$ in $K$ we apply the congruent transformation with

$$
T^{T} K T y=\lambda T^{T} M T y, \quad y=T^{-1} x
$$

where

$$
T=\left(\begin{array}{cccc}
I & O & -K_{11}^{-1} K_{13} & -K_{11}^{-1} K_{14} \\
O & I & -K_{22}^{-1} K_{23} & -K_{22}^{-1} K_{24} \\
O & O & I & O \\
O & O & O & I
\end{array}\right)
$$

Then problem (5) is equivalent to

$$
\left(\begin{array}{cccc}
K_{11} & O & O & O \\
O & K_{22} & O & O \\
O & O & \tilde{K}_{33} & \tilde{K}_{34} \\
O & O & \tilde{K}_{34}^{T} & \tilde{K}_{44}
\end{array}\right)\left(\begin{array}{l}
y_{1} \\
y_{2} \\
y_{3} \\
y_{4}
\end{array}\right)=\lambda\left(\begin{array}{cccc}
M_{11} & O & \tilde{M}_{13} & \tilde{M}_{14} \\
O & M_{22} & \tilde{M}_{23} & \tilde{M}_{24} \\
\tilde{M}_{13}^{T} & \tilde{M}_{23}^{T} & \tilde{M}_{33} & \tilde{M}_{34} \\
\tilde{M}_{14}^{T} & \tilde{M}_{24}^{T} & \tilde{M}_{34}^{T} & \tilde{M}_{44}
\end{array}\right)\left(\begin{array}{l}
y_{1} \\
y_{2} \\
y_{3} \\
y_{4}
\end{array}\right)
$$

where

$$
\begin{aligned}
\tilde{K}_{j j} & =K_{j j}-\sum_{i=1,2} K_{i j}^{T} K_{i i}^{-1} K_{i j}, j=3,4 \\
\tilde{K}_{34} & =K_{34}-\sum_{i=1,2} K_{i 3}^{T} K_{i i}^{-1} K_{i 4} \\
\tilde{M}_{i j} & =M_{i j}-M_{i i} K_{i i}^{-1} K_{i j}, i=1,2, j=3,4 \\
\tilde{M}_{j j} & =M_{j j}-\sum_{i=1,2}\left(M_{i j}^{T} K_{i i}^{-1} K_{i j}-K_{i j}^{T} K_{i i}^{-1} M_{i j}-K_{i j}^{T} K_{i i}^{-1} M_{i i} K_{i i}^{-1} K_{i j}\right), j=3,4 \\
\tilde{M}_{34} & =M_{34}-\sum_{i=1,2}\left(M_{i 3}^{T} K_{i i}^{-1} K_{i 4}-K_{i 3}^{T} K_{i i}^{-1} M_{i 4}-K_{i 3}^{T} K_{i i}^{-1} M_{i i} K_{i i}^{-1} K_{i 4}\right) .
\end{aligned}
$$

Taking advantage of the modal representation

$$
K_{j j} \Phi_{j}=M_{j j} \Phi_{j} \Omega_{j}, \quad \Phi_{j}^{T} M_{j j} \Phi_{j}=I, \quad j=1,2,
$$

of the substructures under consideration, and applying the further congruent transformation by the block diagonal matrix $\tilde{T}=\operatorname{diag}\left\{\Phi_{1}, \Phi_{2}, I, I\right\}$ we obtain the equivalent eigenvalue problem

$$
\left(\begin{array}{cccc}
\Omega_{1} & O & O & O \\
O & \Omega_{2} & O & O \\
O & O & \tilde{K}_{33} & \tilde{K}_{34} \\
O & O & \tilde{K}_{34}^{T} & \tilde{K}_{44}
\end{array}\right)\left(\begin{array}{c}
z_{1} \\
z_{2} \\
z_{3} \\
z_{4}
\end{array}\right)=\lambda\left(\begin{array}{cccc}
I & O & \hat{M}_{13} & \hat{M}_{14} \\
O & I & \hat{M}_{23} & \hat{M}_{24} \\
\hat{M}_{13}^{T} & \hat{M}_{23}^{T} & \tilde{M}_{33} & \tilde{M}_{34} \\
\hat{M}_{14}^{T} & \hat{M}_{24}^{T} & \tilde{M}_{34}^{T} & \tilde{M}_{44}
\end{array}\right)\left(\begin{array}{c}
z_{1} \\
z_{2} \\
z_{3} \\
z_{4}
\end{array}\right),
$$


where

$$
\hat{M}_{i j}=\Phi_{i}^{T} \tilde{M}_{i j}, i=1,2, j=3,4 .
$$

These transformations are applied to all substructures on the finest level. The resulting eigenproblem is a quasistatic-modal representation of the structure which is known as Craigh-Bampton form in component mode synthesis method.

It is well known that the high frequency modes of the substructures do not influence the low frequency modes of the entire structure very much. Hence, similarly as in the component mode synthesis method we can reduce the dimension of the eigenvalue problem (9) considerably if we delete rows and columns which correspond to frequencies of the substructures exceeding a given cut-off frequency.

In a recent paper Bai and Lia [1] suggested a different choice of dropping rows and columns based on a moment-matching analysis. Examples demonstrate that the approximation properties can be improved slightly, usually however, the extra cost do not pay. Moreover, its generalization to several levels is not obvious.

The resulting eigenproblem is the projection of problem (5) to the subspace spanned by all interface degrees of freedom and the kept modes of the substructures of the finest substructuring. It obtains the same block form as problem (9), although the two leading blocks are of much smaller dimension.

Once substructures on the lowest level have been transformed and projected they are assembled to parent substructures on the next level. Again interface and local degrees of freedom are identified, and the substructure models are transformed similarly as on the lowest level.

For instance, for the parent substructure consisting of the three leading block rows and columns of (9) we remove the off-diagonal block $\tilde{K}_{34}$ by a congruent transformation similar to (7) yielding

$$
\left(\begin{array}{cccc}
\Omega_{1} & O & O & O \\
O & \Omega_{2} & O & O \\
O & O & \tilde{K}_{33} & O \\
O & O & O & \hat{K}_{44}
\end{array}\right)\left(\begin{array}{c}
w_{1} \\
w_{2} \\
w_{3} \\
w_{4}
\end{array}\right)=\lambda\left(\begin{array}{cccc}
I & O & \hat{M}_{13} & \bar{M}_{14} \\
O & I & \hat{M}_{23} & \bar{M}_{24} \\
\hat{M}_{13}^{T} & \hat{M}_{23}^{T} & \tilde{M}_{33} & \bar{M}_{34} \\
\bar{M}_{14}^{T} & \bar{M}_{24}^{T} & \bar{M}_{34}^{T} & \bar{M}_{44}
\end{array}\right)\left(\begin{array}{c}
w_{1} \\
w_{2} \\
w_{3} \\
w_{4}
\end{array}\right)
$$

with

$$
\begin{aligned}
& \bar{M}_{i 4}=\hat{M}_{i 4}-\hat{M}_{i 3} \tilde{K}_{33}^{-1} \tilde{K}_{34}, i=1,2,3 \\
& \bar{M}_{44}=\tilde{M}_{44}-\tilde{M}_{34}^{T} \tilde{K}_{33}^{-1} \tilde{K}_{34}-\tilde{K}_{34}^{T} \tilde{K}_{33}^{-1} \tilde{M}_{34}+\tilde{K}_{34}^{T} \tilde{K}_{33}^{-1} \tilde{M}_{33} \tilde{K}_{33}^{-1} \tilde{K}_{34} .
\end{aligned}
$$

Instead of solving the eigenvalue problem corresponding to the local degrees of freedom of the parent substructure under consideration

$$
\left(\begin{array}{ccc}
\Omega_{1} & O & O \\
O & \Omega_{2} & O \\
O & O & \tilde{K}_{33}
\end{array}\right)\left(\begin{array}{l}
v_{1} \\
v_{2} \\
v_{3}
\end{array}\right)=\omega\left(\begin{array}{ccc}
I & O & \hat{M}_{13} \\
O & I & \hat{M}_{23} \\
\hat{M}_{13}^{T} & \hat{M}_{23}^{T} & \tilde{M}_{33}
\end{array}\right)\left(\begin{array}{l}
v_{1} \\
v_{2} \\
v_{3}
\end{array}\right) .
$$

we only take advantage of the eigenproblem of the diagonal block

$$
\tilde{K}_{33} \Phi_{3}=\tilde{M}_{33} \Phi_{3} \Omega_{3}, \Phi_{3}^{T} \tilde{M}_{33} \Phi_{3}=I .
$$




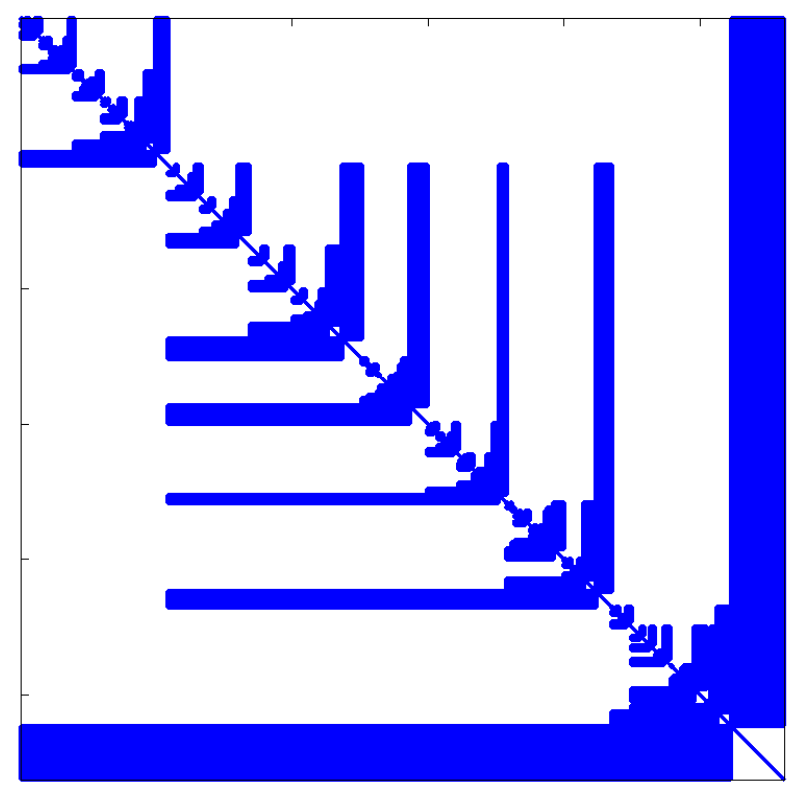

Fig. 2: Transformed mass matrix

Applying the congruent transformation with $T=\operatorname{diag}\left\{I, I, \Phi_{3}, I\right\}$ to problem (9), and dropping all rows and columns in the third block if the corresponding eigenvalue exceeds the cut-off frequency we further reduce the dimension of the eigenproblem.

Assembly to higher-level substructures, transformation to quasistatic-modal representation, and projection to subspaces spanned by modes of diagonal blocks not exceeding the cut-off frequency continues, until we arrive at an approximate model for the entire structure. We end up with a projected eigenproblem

$$
\mathcal{K} y=\lambda \mathcal{M} y,
$$

where the stiffness matrix $\mathcal{K}$ has become diagonal, and the mass matrix is projected to a matrix $\mathcal{M}$ the diagonal of which is the identity, and the only off-diagonal blocks containing non-zero elements are the ones describing the coupling of the substructures and its interfaces. Fig. 2 shows the structure of the resulting mass matrix.

Once the multi-level substructuring transformation of the problem (5) has been accomplished, the substructure modes can be collected in a matrix $\Phi_{\mathrm{AMLS}}$, and if $(\lambda, y)$ is an eigenpair of the projected problem (13) then $(\lambda, x)=\left(\lambda, \Phi_{\text {AмLs }} y\right)$ is an approximate eigenpair of problem (5) called Ritz-pair.

The cost of performing the projection above consists of the cost of obtaining the submatrices $\Phi_{j}$ of $\Phi_{\text {AMLS }}$, and transforming the substructure stiffness and mass matrices $K$ and $M$. Notice that for every substructure only a partial eigenproblem has to be solved, and only a small number of eigenpairs is needed. Moreover, the eigenproblems are usually very small because most of the local degrees of freedom of a substructure are local degrees of the substructures of the next lower level which form the current substructure. Hence, the part of the substructure stiffness matrix corresponding to these degrees of freedom is already diagonal, and we only consider those local degrees of 
freedom which did not have this property on the next lower level, i.e. those interface degrees of freedom of the next lower level which are not interface degrees of freedom on the current level.

Differently from the iterative projection methods like Lanczos, rational Krylov or Jacobi-Davidson where approximations to the wanted eigenpairs are obtained from projections of problem (5) to subspaces which are expanded in the course of the algorithm, AMLS is a one shot method, i.e. after having chosen a cut-off frequency or another dropping rule the method produces one fixed subspace $V$ and the corresponding projected eigenproblem. If the computed approximate eigenpairs turn out to be not accurate enough there is no way to expand the subspace $V$ reusing the projected problem but one has to repeat the reduction with a higher cut-off frequency.

Bekas and Saad [2] identified the level-one version of AMLS as linearization of a rational eigenproblem, which motivated them to suggest three modifications, a second order approximation, expanding the projection space by Krylov subspaces, and a combination of these two modifications. For the multi-level substructuring method, however, these modifications do not seem to be useful.

In a recent paper Yang et al. [29] considered the component mode synthesis method, and they obtained a simple heuristic for choosing spectral components from each substructure suggesting to drop all eigenpairs $(\omega, \phi)$ of substructures in the reduction process such that

$$
\rho_{1}(\omega):=\frac{\lambda_{1}}{\omega-\lambda_{1}} \leq \tau
$$

where $\lambda_{1}$ is the smallest eigenvalue of the problem under consideration, and $\tau$ is a given tolerance.

Taking advantage of a minmax characterization of eigenvalues of nonlinear eigenproblems in [10] we proved that this is an a priori bound to the smallest eigenvalue of problem (5). More generally we proved the following theorem:

THEOREM 1 Let $0<\lambda_{1} \leq \lambda_{2} \leq \cdots \leq \lambda_{n}$ be the eigenvalues of problem (5), and let

$$
\tilde{\lambda}_{1} \leq \tilde{\lambda}_{2} \leq \tilde{\lambda}_{m}<\min _{\nu=0, \ldots, p} \omega_{\nu} \leq \tilde{\lambda}_{m+1} \leq \ldots
$$

be the eigenvalues of the projected eigenproblem by AMLS with $p$ levels of substructuring where on the $\nu$-th level eigenvalues exceeding $\omega_{\nu}$ are neglected. Then it holds

$$
\frac{\tilde{\lambda}_{j}-\lambda_{j}}{\lambda_{j}} \leq \prod_{\nu=0}^{p}\left(1+\frac{\lambda_{j}^{(\nu)}}{\omega_{\nu}-\lambda_{j}^{(\nu)}}\right)-1, \quad j=1, \ldots, m .
$$

Numerical examples demonstrate that these a priori bounds overestimate the real relative errors by a factor up to $10^{2}$. They suggest the following cut-off rule for frequencies: if one is interested to obtain the eigenvalues less than $\hat{\omega}$ with relative errors not exceeding $1 \%$ then a cut-off frequency $10 \hat{\omega}$ usually suffices. 


\section{AMLS for nonlinear eigenproblems}

In this section we consider the nonlinear eigenvalue problem

$$
T(\lambda) x=0
$$

where $T(\lambda) \in \mathbb{C}^{n \times n}$ is a large and sparse matrix depending on a parameter $\lambda \in D \subset$ $\mathbb{C}$. As in the linear case $\lambda \in D$ is an eigenvalue of problem (15), if there exists a nontrivial solution $x \neq 0$ of (15) which we call a corresponding eigenvector.

To generalize the AMLS method we identify an essential linear part of $T(\cdot)$, i.e. we rewrite problem (15) as

$$
K x-\lambda M x-R(\lambda) x=0,
$$

where $K \in \mathbb{C}^{n \times n}$ and $M \in \mathbb{C}^{n \times n}$ are Hermitian and positive definite matrices, and

$$
R(\lambda)=K-\lambda M-T(\lambda)
$$

is a perturbation of the linear eigenproblem $K x=\lambda M x$, which is small in the eigenparameter set of interest.

Once the multi-level substructuring transformation of the linear pencil $(K, M)$ has been accomplished with a given cut-off frequency we obtain a matrix $\Phi_{\text {AMLS }}$ of substructure modes, and a projected eigenproblem

$$
\mathcal{K} y=\lambda \mathcal{M} y
$$

of much smaller dimension where $\mathcal{K}=\Phi_{\mathrm{AMLS}}^{H} K \Phi_{\mathrm{AMLS}}$ and $\mathcal{M}=\Phi_{\mathrm{AMLS}}^{H} M \Phi_{\mathrm{AMLS}}$.

This information can be used in two ways to solve the nonlinear eigenvalue problem approximately: First, we may project the nonlinear eigenproblem (15) to the subspace of $\mathbb{C}^{n}$ spanned by substructure modes which were kept in the AMLS reduction, i.e.

$$
\Phi_{\mathrm{AMLS}}^{H} T(\lambda) \Phi_{\mathrm{AMLS}} y=\mathcal{K} y-\lambda \mathcal{M} y-\Phi_{\mathrm{AMLS}}^{H} R(\lambda) \Phi_{\mathrm{AMLS}} y=0 .
$$

In particular this projection can be performed easily if the remainder $R(\lambda)$ has the form

$$
R(\lambda)=\sum_{j=1}^{p} f_{j}(\lambda) C_{j}
$$

where $f_{j}(\lambda)$ are given complex functions and $C_{j} \in \mathbb{C}^{n \times n}$ are given matrices, which quite often have the same sparsity structure as the pencil $(K, M)$ or some other simple structure. In this case the projection $\Phi_{\mathrm{AMLS}}^{H} R(\lambda) \Phi_{\mathrm{AMLS}}$ could be determined simultaneously with the matrices $\mathcal{K}$ and $\mathcal{M}$ in the course of the AMLS reduction.

Secondly, we may determine Ritz pairs $\left(\lambda_{j}, \Phi_{\text {AмLs }} y_{j}\right), j=1, \ldots, m$ of the linear problem $K x=\lambda M x$ corresponding to eigenvalues in the wanted region from the projected problem (18), and project the nonlinear problem to the subspace spanned by these Ritz vectors. Thus we get

$$
X^{H} T(\lambda) X z=\Lambda z-\lambda z-X^{H} R(\lambda) X z=0
$$


where $\Lambda=\operatorname{diag}\left\{\lambda_{1}, \ldots, \lambda_{m}\right\}$ and $X=\left(x_{1}, \ldots, x_{m}\right)$.

Problem (20) is equivalent to the projection of problem (19) to the space spanned by the eigenvectors $y_{1}, \ldots, y_{m}$ of (18) corresponding to $\lambda_{1}, \ldots, \lambda_{m}$. Hence, we can expect, that the first approach will yield better approximations. Examples, however, demonstrate that the loss of accuracy is often negligible.

In either case we arrive at a projected nonlinear eigenvalue problem of much smaller dimension which can be solved by a dense solver or an iterative projection method depending on the dimension. The following subsections demonstrate how AMLS can be applied to some classes of nonlinear eigenproblems successfully.

\subsection{Gyroscopic problems}

Consider the gyroscopic eigenvalue problem

$$
Q(\omega) x:=K x+i \omega G x-\omega^{2} M x=0
$$

governing eigenvibrations of rotating structures. Here $K$ is the stiffness matrix modified by the presence of centripetal forces, $M$ is the mass matrix, and $G$ is the gyroscopic matrix stemming from the Coriolis force. Clearly, $K$ and $M$ are symmetric and positive definite, and $G$ is skew-symmetric.

For example, problem (21) arises when modeling noise of rolling tires which is the major source of traffic noise for passenger cars at speed exceeding $60 \mathrm{~km} / \mathrm{h}$ (cf. $[19,20])$. Due to the complicated interior structure of a belted tire the matrices $K$, $M$ and $G$ of a sufficiently accurate FE model are very large and sparse. Moreover, for the acoustic analysis many eigenpairs not necessarily at the end of the spectrum are needed. Therefore, well-established sparse eigensolvers of Arnoldi type with shift and invert techniques [18] for a linearization of problem (21) or iterative projection methods for nonlinear eigenproblems [28] are very costly since LU factorizations of complex valued matrices $Q\left(\omega_{j}\right)$ for several parameters $\omega_{j}$ are required.

Since the influence of the gyroscopic matrix $G$ on the eigenvalues of (21) is usually not very high compared to the mass and stiffness matrix, it is reasonable to neglect the linear term $i \omega G$ when defining the essential linear eigenproblem $K x=\omega^{2} M x$.

Since the sparsity pattern of $G$ matches the ones of $K$ and $M$ one gets the reduced model

$$
\mathcal{K} y+i \omega \mathcal{G} y-\omega^{2} \mathcal{M} y=0,
$$

when applying the AMLS reduction to $K x=\omega^{2} M x$, and projecting the matrix $G$ simultaneously. Here the stiffness and mass matrix have the same structure as in the linear case, and the gyroscopic matrix $\mathcal{G}$ is a skew-symmetric block matrix containing diagonal blocks corresponding to the (reduced) substructures and interfaces, and only off-diagonal blocks describing the coupling of a substructure and its interface contain non-zero elements. Notice, that all projectors are real, and therefore the reduction can be performed in real arithmetic. 
If the dimension of problem (22) is very small, a method at hand is to consider the linearization

$$
\left(\begin{array}{cc}
i \mathcal{G} & \mathcal{K} \\
\mathcal{K} & O
\end{array}\right)\left(\begin{array}{c}
\omega y \\
y
\end{array}\right)=\omega\left(\begin{array}{cc}
\mathcal{M} & O \\
O & \mathcal{K}
\end{array}\right)\left(\begin{array}{c}
\omega y \\
y
\end{array}\right)
$$

of problem (22) and to apply any dense solver.

For very large gyroscopic problems (for instance a realistic model of a rolling tire) the dimension of the projected problem (22) will still be quite large. In this case (22) can be solved by an iterative projection method taking advantage of the minmax characterization of its positive eigenvalues [23] or by a sparse solver of (23) like ARPACK [18]. In both cases the solution requires complex arithmetic.

As a numerical example we consider a tire model with 39204 brick elements, 124992 degrees of freedom and 20 different material groups, rotating with $50 \mathrm{~km} / \mathrm{h}$. Our aim is to determine approximations to the smallest 180 eigenvalues with relative error less than $1 \%$ and the corresponding eigenvectors.

Linearizing problem (21) in the usual way

$$
\left(\begin{array}{cc}
-i G & -K \\
I & O
\end{array}\right)\left(\begin{array}{c}
\omega x \\
x
\end{array}\right)=\omega\left(\begin{array}{cc}
M & O \\
O & I
\end{array}\right)\left(\begin{array}{c}
\omega x \\
x
\end{array}\right)
$$

or by the Hermitian problem

$$
\left(\begin{array}{cc}
i G & K \\
K & O
\end{array}\right)\left(\begin{array}{c}
\omega x \\
x
\end{array}\right)=\omega\left(\begin{array}{cc}
M & O \\
O & K
\end{array}\right)\left(\begin{array}{c}
\omega x \\
x
\end{array}\right)
$$

and applying the shift-and-invert Arnoldi method requires an LU factorization of $Q(\omega)$ for every shift $\omega$, which is a complex matrix. Determining the factorization by SuperLU [9] requires a memory of 6.04 GByte and a CPU time of 3910 seconds on one PA-RISC $(750 \mathrm{MHz})$ processor of an HP superdome.

Applying the nonlinear Arnoldi method [23] the preconditioners can be chosen as real matrices $K-\omega^{2} M$, the LU factorization of which requires 2.7 GByte storage and 1940 seconds with SuperLU, and 2.86 GByte storage and 1080 seconds with the multi frontal solver MA57 of HSL [14]. Since the LU factorization has to be updated several times a total CPU time of more than 12 hours results on one processor of the superdome.

AMLS demands much less storage and the problem under consideration can be solved on a personal computer, namely a Pentium 4 processor with $3.0 \mathrm{GHz}$ and 1 GByte storage. With a cut-off frequency of $\omega_{c}=2 \times 10^{5}$ the problem is projected to a gyroscopic eigenproblem (22) of dimension $n_{c}=2697$ requiring a CPU time of 1187 seconds. Solving the linearization (23) of the projected problem (22) by eigs (i.e. by ARPACK) under MATLAB 7.0 requires another 166.1 seconds.

Figure 3 shows the relative errors of all 180 eigenvalues which are all less than $0.67 \%$. Results from the nonlinear Arnoldi method are used as reference values.

Projecting problem (21) to the subspace spanned by the Ritz vectors corresponding to eigenvalues of the linear problem

$$
\mathcal{K} y=\lambda \mathcal{M} y
$$




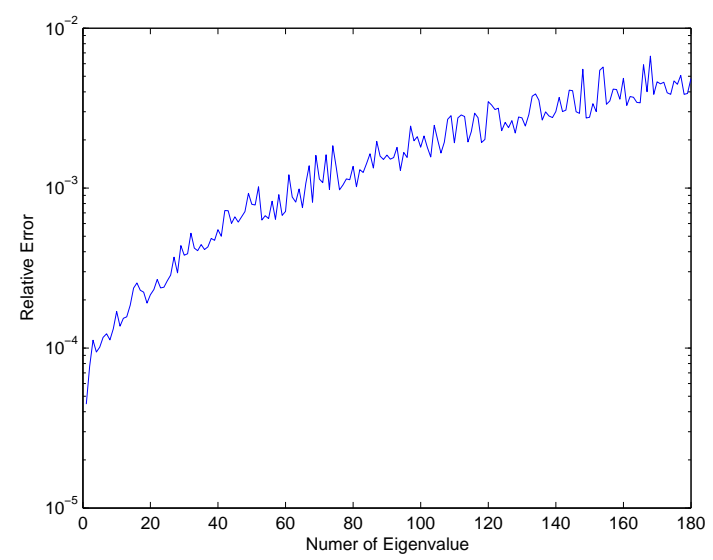

Fig. 3: Relative errors of smallest 180 eigenvalues

not exceeding $1.5 \omega_{\max }^{2}$ where $\omega_{\max }=12000$ is the maximal wanted eigenvalue one gets a gyroscopic eigenproblem of dimension 262. The accuracy of the approximations to the 180 smallest eigenvalues of (21) is deteriorated only slightly. The maximum relative error is raised only to $0.69 \%$.

Numerically this can be done by solving problem (26) by a sparse linear solver (eigs, e.g.), projecting problem (22) to the invariant subspace corresponding to the chosen eigenvalues, and solving a linearization of the projected problem by a dense solver. This way the computing cost is decreased to 30.5 seconds to solve (26), 5.2 seconds to obtain the projected problem, and 3.9 seconds to solve it.

\subsection{Nonproportional damping}

We consider a finite element model of a vibrating structure with nonproportional damping. Using a viscoelastic constitutive relation to describe the behavior of a material in the equations of motions yields a rational eigenvalue problem for the case of free vibrations [12]. A finite element model obtains the form

$$
T(\omega) x:=\left(\omega^{2} M+K-\sum_{j=1}^{J} \frac{1}{1+b_{j} \omega} \Delta K_{j}\right) x=0
$$

where $M$ is the consistent mass matrix, $K$ is the stiffness matrix with the instantaneous elastic material parameters used in Hooke's law, $J$ denotes the number of regions with different relaxation parameters $b_{j}$, and $\Delta K_{j}$ is an assemblage of element stiffness matrices over the region with the distinct relaxation constants. The real part of an eigenvalue is the exponential rate with which the motion described by the corresponding eigenvector $x$ decays. The imaginary part is the (damped) angular frequency with which the motion described by $x$ oscillates.

We consider the feeder clamp in Fig. 4 from the model library of FEMLAB [11] which is clamped at its bottom. The instantaneous Young's modulus is set to $E=$ 

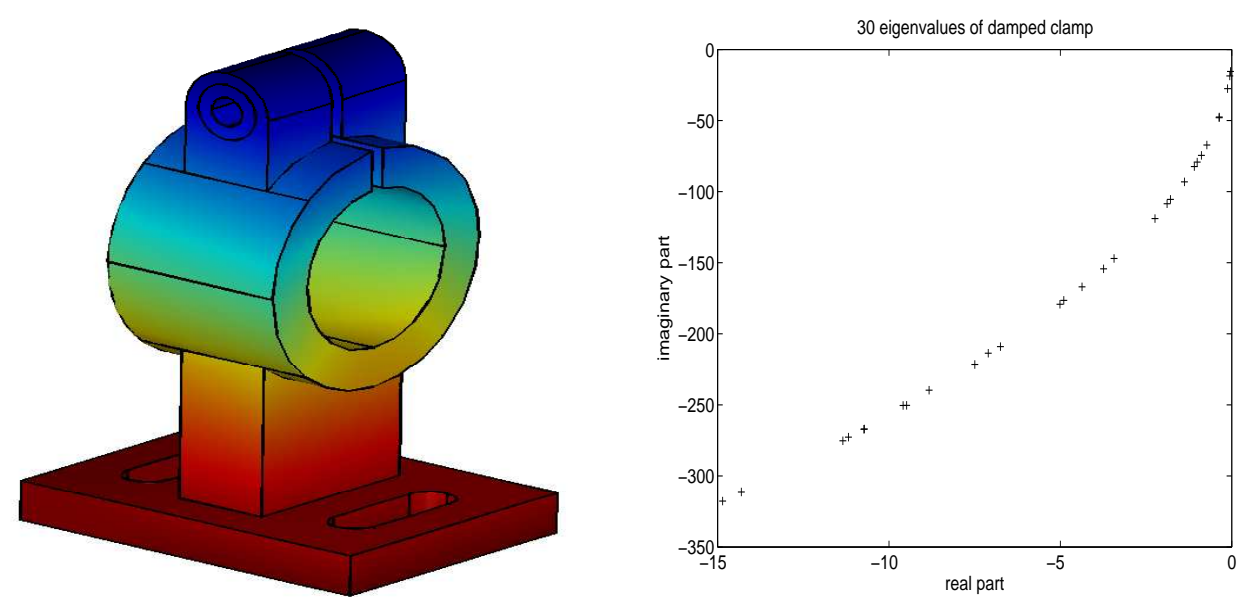

Fig. 4: feeder clamp / eigenvalues

$2.06 \times 10^{11}$, the instantaneous Poisson's rate is $\nu=0.3$, and the density is set to $\rho=$ 7800. For the nonproportional damping we use in addition the following parameters, $\Delta \nu=0.28$, and $\Delta E=6 \times 10^{10}$, and the relaxation constant is set to $b=1 \times 10^{-3}$.

Discretizing this problem by linear Lagrangian elements we obtained the rational eigenproblem (27) of dimension 193617. For symmetry reasons we determined only eigenvalues with negative imaginary part, and we computed 30 of them one after another with decreasing imaginary part. We solved this problem bei the nonlinear Arnoldi method [26] where we preconditioned by an incomplete LU decomposition and accepted an eigenpair if the residual was less than $10^{-4}$. This required a CPU time of 8309 seconds on an AMD Opteron Processor 248 x860_64 with $2.2 \mathrm{GHz}$ and 4 GB RAM under MATLAB 7.0.

Applying the AMLS method to the linear eigenvalue problem

$$
K x=\lambda M x
$$

with cut-off frequency $\lambda_{c}=1.2 e 7$ and $\lambda_{c}=2.4 e 7$, respectively, and applying the transformations and projections to the matrix $\Delta K$ simultaneously, we obtained an eigenvalue problem

$$
\left(\omega^{2} \mathcal{M}+\mathcal{K}-\frac{1}{1+b \omega} \Delta \mathcal{K}\right) y=0
$$

of dimension $d_{1}=1262$ and $d_{2}=2808$, which required 837 and 996 seconds, respectively.

Multiplying (29) by $1+b \omega$ one gets a polynomial eigenvalue problem of degree 3 which is equivalent to the linearized eigenvalue problem

$$
\left(\begin{array}{ccc}
O & I & O \\
O & O & I \\
\Delta \mathcal{K}-\mathcal{K} & -b \mathcal{K} & -\mathcal{M}
\end{array}\right)\left(\begin{array}{c}
y \\
\omega y \\
\omega^{2} y
\end{array}\right)=\omega\left(\begin{array}{ccc}
I & O & O \\
O & I & O \\
O & O & b \mathcal{M}
\end{array}\right)\left(\begin{array}{c}
y \\
\omega y \\
\omega^{2} y
\end{array}\right)
$$

of dimension $3 d_{j}, j \in\{1,2\}$. Approximations to the desired 30 eigenvalues of (27) can be obtained from problem (30) by the MATLAB function eigs (i.e. by ARPACK) 


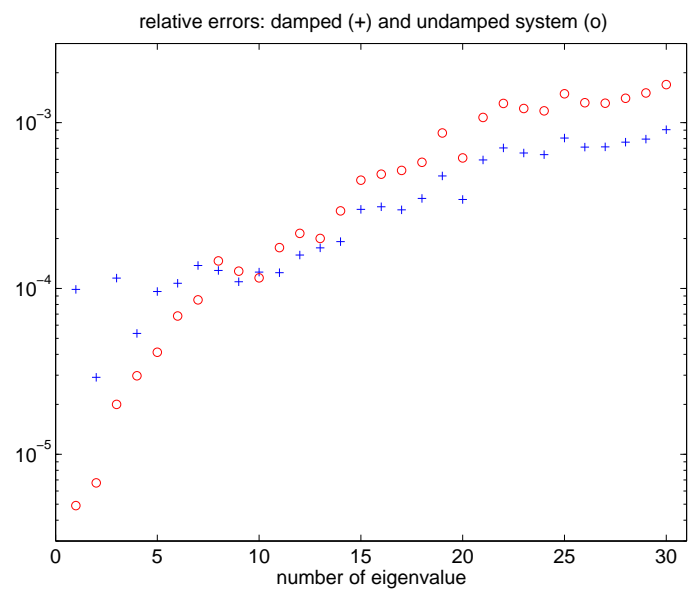

Fig. 5: Relative errors of 30 eigenvalues

requiring 7.1 and 24.2 seconds, respectively. The maximum relative error is $2.1 e-3$ for the projected problem of dimension 1262 and $9.1 e-4$ for the problem of dimension 2808. Fig. 5 shows the relative errors of the AMLS method for the linear eigenproblem (28) marked by circles and the rational eigenproblem (27) marked by plus signs.

The solution time for the projected problem can be further reduced to 4.7 and 5.6 seconds, respectively, if we project problem (29) further to the space spanned by the eigenvectors of the linear eigenproblem $\mathcal{K} y=\lambda \mathcal{M} y$ corresponding to eigenvalues not exceeding $\lambda_{c}=1.5\left|\tilde{\omega}_{p}\right|^{2}$. This approach obviously is equivalent to determining Ritz vectors of the linear problem $K x=\lambda M x$ corresponding to eigenvalues not exceeding $\lambda_{c}$ and projecting problem (27) to the space spanned by these Ritz vectors. The maximum relative error is raised only slightly to $9.3 e-4$.

\subsection{Vibrations of fluid-solid structures}

We consider free vibrations of a tube bundle immersed in a slightly compressible fluid under the following simplifying assumptions: The tubes are assumed to be rigid, assembled in parallel inside the fluid, and elastically mounted in such a way that they can vibrate transversally, but they can not move in the direction perpendicular to their sections. The fluid is assumed to be contained in a cavity which is infinitely long, and each tube is supported by an independent system of springs (which simulates the specific elasticity of each tube). Due to these assumptions, three-dimensional effects are neglected, and so the problem can be studied in any transversal section of the cavity. Considering small vibrations of the fluid (and the tubes) around the state of rest, it can also be assumed that the fluid is irrotational.

Mathematically this problem can be described in the following way (cf. [7, 8, 21]). Let $\Omega \subset \mathbb{R}^{2}$ (the section of the cavity) be an open bounded set with locally Lipschitz continuous boundary $\Gamma$. We assume that there exists a family $\Omega_{j} \neq \emptyset, j=1, \ldots, p$, (the sections of the tubes) of simply connected open sets such that $\bar{\Omega}_{j} \subset \Omega$ for every $j, \bar{\Omega}_{j} \cap \bar{\Omega}_{i}=\emptyset$ for $j \neq i$, and each $\Omega_{j}$ has a locally Lipschitz continuous boundary $\Gamma_{j}$. 
With these notations we set $\Omega_{0}:=\Omega \backslash \bigcup_{j=1}^{p} \bar{\Omega}_{j}$. Then the boundary of $\Omega_{0}$ consists of $p+1$ connected components which are $\Gamma$ and $\Gamma_{j}, j=1, \ldots, p$.

We denote by $H^{1}\left(\Omega_{0}\right)=\left\{u \in L^{2}\left(\Omega_{0}\right): \nabla u \in L^{2}\left(\Omega_{0}\right)^{2}\right\}$ the standard Sobolev space, and consider the subspace

$$
H:=\left\{u \in H^{1}\left(\Omega_{0}\right): \int_{\Omega_{0}} u(x) d x=0\right\}
$$

equipped with the usual scalar product

$$
\langle u, v\rangle:=\int_{\Omega_{0}} \nabla u(x) \cdot \nabla v(x) d x .
$$

Then the eigenfrequencies and the eigenmodes of the fluid-solid structure are governed by the following variational eigenvalue problem (cf. [8])

Find $\lambda \in \mathbb{R}$ and $u \in H$ such that for every $v \in H$

$$
c^{2} \int_{\Omega_{0}} \nabla u \cdot \nabla v d x=\lambda \int_{\Omega_{0}} u v d x+\sum_{j=1}^{p} \frac{\lambda \rho_{0}}{k_{j}-\lambda m_{j}} \int_{\Gamma_{j}} u n d s \cdot \int_{\Gamma_{j}} v n d s .
$$

Here $u$ is the potential of the velocity of the fluid, $c$ denotes the speed of sound in the fluid, $\rho_{0}$ is the specific density of the fluid, $k_{j}$ represents the stiffness constant of the spring system supporting tube $j, m_{j}$ is the mass per unit length of the tube $j$, and $n$ is the outward unit normal on the boundary of $\Omega_{0}$.

The eigenvalue problem is non-standard in two respects: The eigenparameter $\lambda$ appears in a rational way in the boundary conditions, and the boundary conditions are nonlocal. Using methods from linear functional analysis Conca, Planchard and Vanninathan $[7,8]$ proved that this problem has a countable set of eigenvalues which are positive and real and which converge to infinity. To this end they transformed the rational eigenproblem to a linear compact eigenproblem on a Hilbert space which is nonselfadjoint but can be symmetrized easily. The same result follows more easily from the fact that the eigenvalues of of problem (31) can be characterized as minmax values of a Rayleigh functional [25].

In particular we consider the rational eigenvalue problem (31) where $\Omega$ is the ellipse with center $(0,0)$ and length of semiaxes 8 and 4 , and $\Omega_{j}, j=1, \ldots, 9$ are circles with radius 0.3 and centers $(-4,-2),(0,-2),(4,-2),(-5,0),(0,0),(5,0),(-4,2)$, $(0,2)$ and $(4,2)$. We assume that $c=1, \rho_{0}=1$ and $m_{j}=1$ for all $j$. For the stiffness constants $k_{j}$ we assume $k_{1}=k_{2}=k_{3}=1, k_{4}=k_{5}=k_{6}=2$, and $k_{7}=k_{8}=k_{9}=3$.

Discretizing problem (31) by linear Lagrangian elements one gets a rational matrix eigenvalue problem

$$
T(\lambda) x:=-K x+\lambda M x+\frac{\lambda}{1-\lambda} C_{1} C_{1}^{T} x+\frac{\lambda}{2-\lambda} C_{2} C_{2}^{T} x+\frac{\lambda}{3-\lambda} C_{3} C_{3}^{T} x=0
$$


where $K$ and $M$ are symmetric and positive (semi-)definite, and $C_{j} \in \mathbb{R}^{n \times 6}, j=$ $1,2,3$, collects the contributions of the three groups of tubes in the nonlocal boundary conditions. In our example the dimension is $n=143064$.

Problem (32) has 18, 15, and 14 eigenvalues in the interval $J_{1}=(0,1), J_{2}=(1,2)$, and $J_{3}=(2,3)$, respectively, and a large number of eigenvalues in $(3, \infty), 18$ of which are contained in $J_{4}:=(3,5)$. In each of the intervals $J_{j}$ the eigenvalues can be characterized as minmax values of a Rayleigh functional [25], and they can be determined one after the other by the nonlinear Arnoldi method [23]. Preconditioning by the LU factorization of $T(\sigma)$ for fixed $\sigma \in J_{j}$ and starting with initial search spaces according to [24] the Arnoldi method required 795 seconds to determine all eigenvalues in $[0,5]$ on an AMD Opteron Processor 248 x86_64 with $2.2 \mathrm{GHz}$ and 4 GB RAM under MATLAB 7.0.

The reduction of the problem by AMLS with the base problem $K x=\lambda M x$ and a cut-off frequency of 100 generated a rational eigenvalue problem

$$
-\mathcal{K} y+\lambda \mathcal{M} y+\frac{\lambda}{1-\lambda} \mathcal{C}_{1} \mathcal{C}_{1}^{T} y+\frac{\lambda}{2-\lambda} \mathcal{C}_{2} \mathcal{C}_{2}^{T} y+\frac{\lambda}{3-\lambda} \mathcal{C}_{3} \mathcal{C}_{3}^{T} y=0
$$

of dimension 888 . In principle this problem could be multiplied by $(1-\lambda)(2-\lambda)(3-\lambda)$ yielding a polynomial eigenvalue problem of degree 4 . Linearization would result in a linear eigenvalue problem of dimension 3552 which could be solved by a sparse solver like ARPACK. However, since $\operatorname{rank}\left(\mathcal{C}_{j}\right)=6, j=1,2,3$, the polynomial eigenproblem and its linearization have eigenvalues $\tilde{\lambda}_{j}=j, j=1,2,3$ each of multiplicity 882 , which would impede the computation of the eigenvalues in the interval $[0,5]$.

Since the projected problem (33) inherits the symmetry properties of problem (32) we solved it by the nonlinear Arnoldi method, which required 451.42 seconds for reducing the base problem $K x=\lambda M x$ by AMLS and transforming the matrices $C_{1}$, $C_{2}$, and $C_{3}$ simultaneously, and 45.64 seconds for solving the projected problem (33) by the nonlinear Arnoldi method. Hence, the total CPU time was 497.06 seconds. The gain in computing time by the AMLS method for this 2 dimensional problem is not as pronounced as in our previous examples since the matrices are less populated, and the preconditioner in the nonlinear Arnoldi method requires much less storage and arithmetic operations .

The relative errors are displayed as plus signs in Fig. 6. The maximum error is 0.063 which is quite large for a cut-off frequency being 20 times larger than the largest wanted eigenvalue. This difficulty is caused by the fact that the rational eigenproblem (32) is not just a small perturbation of the base problem $K x=\lambda M x$ used in the AMLS reduction (for instance, (32) has 18 eigenvalues in the interval $[0,1)$ whereas the linear problem $K x=\lambda M x$ has only 12). There are eigenvectors of problem (32) which have large amplitudes close to some of the tubes which can not be approximated well by the AMLS basis consisting of eigenmodes of interfaces and substructures. Fig. 7 and Fig. 8 show two of these eigenfunctions.

It is interesting to note that these eigenfunctions do not correspond to eigenvalues which are close to a pole. Fig. 9 shows the eigenfunction corresponding to an eigen- 


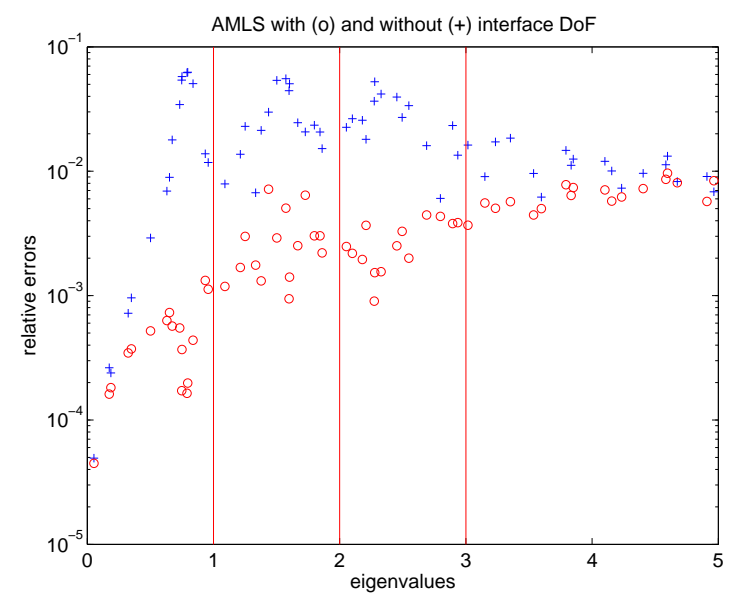

Fig. 6: Relative errors for fluid-solid structure

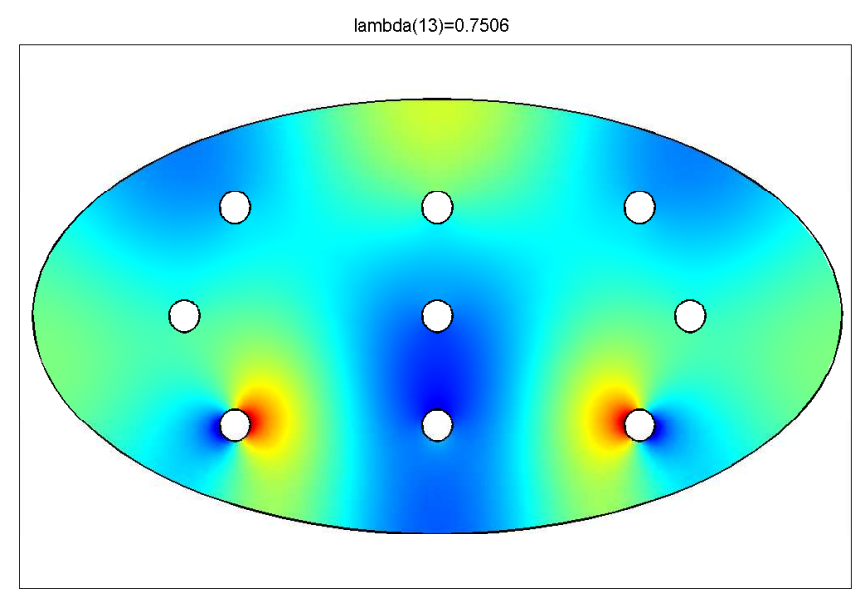

Fig. 7: Eigenvector corresponding to $\lambda_{13}=0.7506$

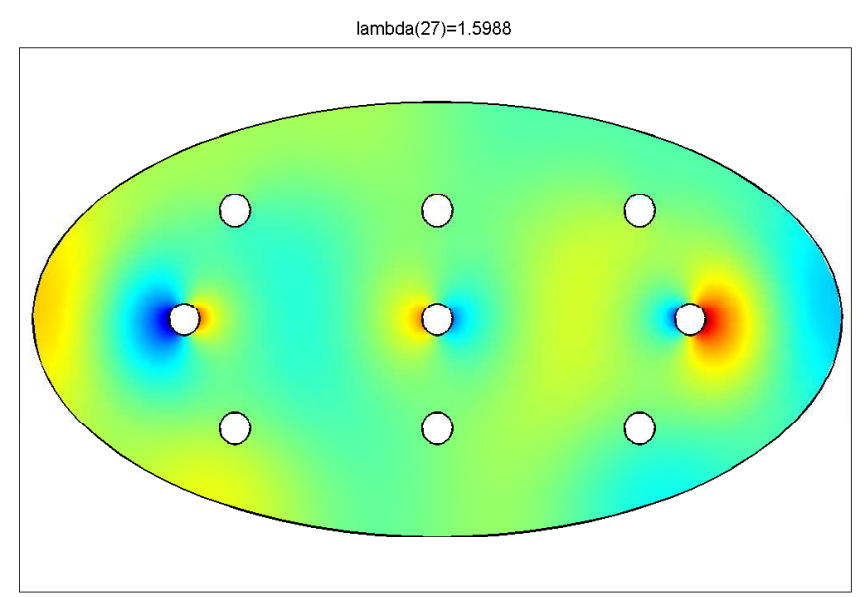

Fig. 8: Eigenvector corresponding to $\lambda_{27}=1.5988$ 


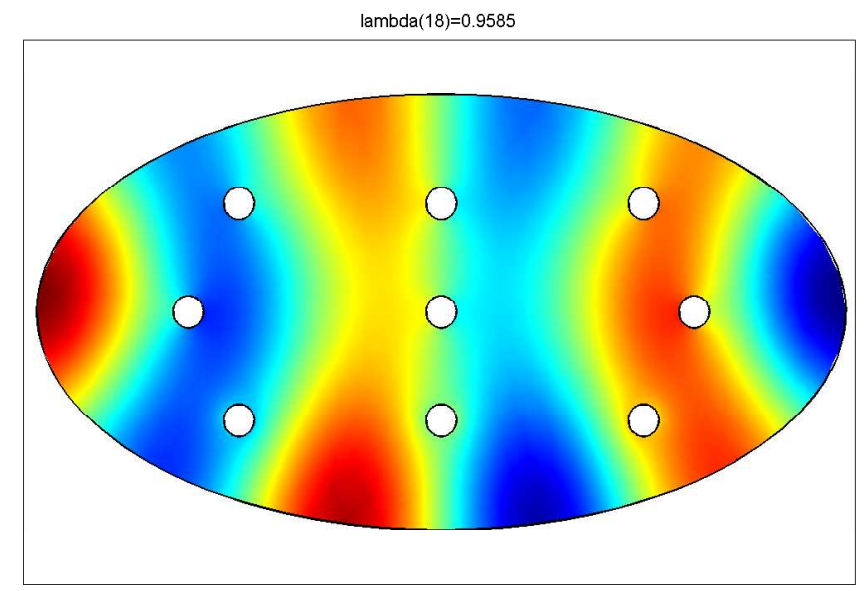

Fig. 9: Eigenvector corresponding to $\lambda_{18}=0.9585$

value $\lambda_{18}$ close to the pole $\sigma_{1}=1$ which can be easily approximated by the base vectors.

To improve the approximation properties of AMLS we complemented the 503 interface degrees of freedom on the coarsest level which were generated by METIS for the base problem $K x=\lambda M x$ by the 1728 components corresponding to nonzero row entries of the matrix $\left[C_{1}, C_{2}, C_{3}\right]$. Applying AMLS with the cut-off frequency 100 we obtained a reduced problem of the type (33) of dimension 902 which we solved by the nonlinear Arnoldi method. The maximum relative error was reduced to 0.0096. The relative errors are displayed in Fig. 6 as circles. The total CPU time was 527.03 seconds, 480.89 for the AMLS reduction, and 46.14 seconds for the solution of the reduced problem.

The alternative way of solving the nonlinear eigenproblem by projecting it to a subspace spanned by a moderate number of Ritz vectors obtained from the AMLS method for the linear base problem leads to bad approximations in this problem. For instance, if problem (32) is projected to the space (of dimension 88) spanned by the Ritz vectors corresponding to the Ritz values not exceeding 10 (the double of the maximal wanted eigenvalue), the maximum relative error for the eigenvalues in the interval $[0,1)$ is 0.15 .

\section{Conclusions}

The Automated Multi-Level Substructuring method (AMLS) is known to be very efficient for huge linear definite eigenvalue problems $K x=\lambda M x$. Here the joint graph of $K$ and $M$ is partitioned into many subgraphs on several levels, and the problem is projected to a subspace spanned by eigenmodes of the matrices corresponding to subgraphs and a hierarchy of interfaces.

In this paper we generalized this approach to nonlinear eigenvalue problems identifying a linear base problem and projecting the underlying problem to the subspace 
generated by applying AMLS to the base problem.

The method works well for nonlinear problems which are small perturbations of a linear definite problem. For problems where the nonlinearity is more pronounced the AMLS method can be improved by complementing the interface degrees of freedom on the coarsest level by further degrees of freedom which are suitable to catch the nonlinear behavior. Numerical methods from structural dynamics demonstrate the efficiency of the method.

\section{Literatur}

[1] Z. Bai and B.-S. Lia. Towards an optimal substructuring method for model reduction. Technical report, University of California at Davis, 2004. To appear in Proceedings of PARA’04, Lyngby, Denmark, 2004.

[2] C. Bekas and Y. Saad. Computation of smallest eigenvalues using spectral Schur complements. Technical Report umsi-2003-191, Minnesota Supercomputer Institute, University of Minnesota, Minneapolis, 2004.

[3] J. Bennighof. Vibroacoustic frequency sweep analysis using automated multilevel substructuring. In Proceedings of the AIAA $40^{\text {th }}$ SDM Conference, St. Louis, Missouri, 1999.

[4] J.K. Bennighof and M.F. Kaplan. Frequency sweep analysis using multi-level substructuring, global modes and iteration. In Proceedings of the AIAA 39th SDM Conference, Long Beach, Ca., 1998.

[5] J.K. Bennighof, M.F. Kaplan, and M.B. Muller. Extending the frequency response capabilities of automated multi-level substructuring. In Proceedings of the $41^{\text {st }}$ AIAA/ASME/ASCE/AHS SDM Conference, number AIAA-2000-1574, 2000 .

[6] J.K. Bennighof and R.B. Lehoucq. An automated multilevel substructuring method for the eigenspace computation in linear elastodynamics. SIAM J. Sci. Comput., 25:2084 - 2106, 2004.

[7] C. Conca, J. Planchard, and M. Vanninathan. Existence and location of eigenvalues for fluid-solid structures. Comput.Meth.Appl.Mech.Engrg., 77:253 - 291, 1989.

[8] C. Conca, J. Planchard, and M. Vanninathan. Fluid and Periodic Structures, volume 24 of Research in Applied Mathematics. Masson, Paris, 1995.

[9] J.W. Demmel, J.R. Gilbert, and X.S. Li. SuperLU Users' Guide. Technical Report LBNL-44289, Lawrence Berkeley National Laboratory, 2003. Available at http://crd.lbl.gov/ xiaoye/SuperLU/. 
[10] K. Elssel and H. Voss. An a priori bound for automated multilevel substructuring. Technical Report 81, Section of Mathematics, Hamburg University of Technology, 2004. Submitted to SIAM J.Matr.Anal.Appl. Available at http: //www.tu-harburg.de/mat/Schriften/rep/rep81.pdf.

[11] FEMLAB, Version 3.1. COMSOL, Inc., Burlington, MA, USA, 2004.

[12] P. Hager and N.E. Wiberg. The rational Krylov algorithm for nonlinear eigenvalue problems. In B.H.V. Topping, editor, Computational Mechanics for the Twenty-First Century, pages 379 - 402. Saxe-Coburg Publications, Edinburgh, 2000.

[13] B. Hendrickson and R. Leland. The Chaco User's Guide: Version 2.0. Technical Report SAND94-2692, Sandia National Laboratories, Albuquerque, 1994.

[14] HSL2002: A catalogue of subroutines, 2002. Available at http://www. aspentech.com/hsl/cat_hsl2002.pdf.

[15] M.F. Kaplan. Implementation of Automated Multilevel Substructuring for Frequency Response Analysis of Structures. PhD thesis, Dept. of Aerospace Engineering \& Engineering Mechanics, University of Texas at Austin, 2001.

[16] G. Karypis and V. Kumar. Metis. a software package for partitioning unstructured graphs, partitioning meshes, and computing fill-reducing orderings of sparse matrices. version 4.0. Technical report, University of Minnesota, Minneapolis, 1998.

[17] A. Kropp and D. Heiserer. Efficient broadband vibro-acoustic analysis of passenger car bodies using an FE-based component mode synthesis approach. J.Comput.Acoustics, 11:139 - 157, 2003.

[18] R.B. Lehoucq, D.C. Sorensen, and C. Yang. ARPACK Users' Guide. Solution of Large-Scale Eigenvalue Problems with Implicitly Restarted Arnoldi Methods. SIAM, Philadelphia, 1998.

[19] U. Nackenhorst. Rollkontaktdynamik - Numerische Analyse der Dynamik rollender Körper mit der Finite Elemente Methode. Habilitationsschrift, Institut für Mechanik, Universität der Bundeswehr, Hamburg, 2000.

[20] U. Nackenhorst and O. von Estorff. Numerical analysis of tire noise radiation - a state of the art review. In Inter-noise 2001. The 2001 International Congress and Exhibition on Noise Control Engineering, The Hague, The Netherlands, 2001.

[21] J. Planchard. Eigenfrequencies of a tube bundle placed in a confined fluid. Comput.Meth.Appl.Mech.Engrg., 30:75 - 93, 1982. 
[22] R. Stryczek, A. Kropp, S. Wegner, and F. Ihlenburg. Vibro-acoustic computations in the mid-frequency range: efficiency,evaluation, and validation. In Proceedings of the International Conference on Noise \& Vibration Engineering, ISMA 2004, KU Leuven, 2004. CD-ROM ISBN 90-73802-82-2.

[23] H. Voss. An Arnoldi method for nonlinear symmetric eigenvalue problems. In Online Proceedings of the SIAM Conference on Applied Linear Algebra, Williamsburg., http://www.siam.org/meetings/laa03/, 2003.

[24] H. Voss. Initializing iterative projection methods for rational symmetric eigenproblems. In Online Proceedings of the Dagstuhl Seminar Theoretical and Computational Aspects of Matrix Algorithms, Schloss Dagstuhl 2003, ftp://ftp.dagstuhl.de/pub/Proceedings/03/03421/03421.VoszHeinrich.Other.pdf, 2003.

[25] H. Voss. A rational spectral problem in fluid-solid vibration. Electronic Transactions on Numerical Analysis, 16:94 - 106, 2003.

[26] H. Voss. An Arnoldi method for nonlinear eigenvalue problems. BIT Numerical Mathematics, 44:387 - 401, 2004.

[27] H. Voss. A Jacobi-Davidson method for nonlinear and nonsymmetric eigenproblems. Technical report, Section of Mathematics, Hamburg University of Technology, 2004. Submitted to Computers \& Structures.

[28] H. Voss. Numerical methods for sparse nonlinear eigenproblems. In Ivo Marek, editor, Proceedings of the XV-th Summer School on Software and Algorithms of Numerical Mathematics, Hejnice, 2003, pages 133 - 160, University of West Bohemia, Pilsen, Czech Republic, 2004. Available at http://www.tu-harburg.de/mat/Schriften/rep/rep70.pdf.

[29] C. Yang, W. Gao, Z. Bai, X. Li, L. Lee, P. Husbands, and E. Ng. An algebraic sub-structuring method for large-scale eigenvalue calculations. Technical Report LBNL-55050, Lawrence Berkeley National Laboratory, Berkeley, Ca., 2004. 